\title{
Free group automorphisms with many fixed points at infinity
}

\author{
ANDRÉ JÄGER \\ MARTIN LUSTIG
}

\begin{abstract}
A concrete family of automorphisms $\alpha_{n}$ of the free group $F_{n}$ is exhibited, for any $n \geq 3$, and the following properties are proved: $\alpha_{n}$ is irreducible with irreducible powers, has trivial fixed subgroup, and has $2 n-1$ attractive as well as $2 n$ repelling fixed points at $\partial F_{n}$. As a consequence of a recent result of $\mathrm{V}$ Guirardel there can not be more fixed points on $\partial F_{n}$, so that this family provides the answer to a question posed by G Levitt.
\end{abstract}

20E36; 57M05

\section{Introduction}

Let $F_{n}$ be a free group of finite rank $n \geq 2$. It is well known that every automorphism $\alpha$ of $F_{n}$ induces a homeomorphism $\partial \alpha$ on the Gromov boundary $\partial F_{n}$. Every fixed point of $\partial \alpha$ is either attracting or repelling (= attracting for $\left.\partial \alpha^{-1}\right)$ or it belongs to $\partial \operatorname{Fix}(\alpha)$, which embedds into $\partial F_{n}$, as the fixed subgroup $\operatorname{Fix}(\alpha)=\left\{w \in F_{n} \mid \alpha(w)=w\right\}$ is quasiconvex in $F_{n}$. Notice that $\operatorname{Fix}(\alpha)$ acts on the set of attracting fixed points $\mathrm{Fix}^{+}(\partial \alpha)$ of $\partial \alpha$. After various proofs that $\operatorname{Fix}(\alpha)$ is finitely generated and that $\operatorname{Fix}^{+}(\partial \alpha) / \operatorname{Fix}(\alpha)$ is finite for all $\alpha \in \operatorname{Aut}\left(F_{n}\right)$ (see Gersten [7], Cooper [3], Goldstein and Turner [8], Cohen and Lustig [2], Paulin [15], Gaboriau, Levitt, and Lustig [6] etc), the following improvement of Bestvina and Handel's Theorem [1] (also known as the the Scott Conjecture) has been given by Gaboriau, Jaeger, Levitt and Lustig [4]:

$$
\operatorname{rk}(\operatorname{Fix}(\alpha))+\frac{1}{2} \#\left(\operatorname{Fix}^{+}(\partial \alpha) / \operatorname{Fix}(\alpha)\right) \leq n
$$

It follows in particular that, if $\operatorname{Fix}(\alpha)$ is trivial, then the total set of fixed points $\operatorname{Fix}(\partial \alpha)=\operatorname{Fix}^{+}(\partial \alpha) \cup \operatorname{Fix}^{+}\left(\partial \alpha^{-1}\right)$ at $\partial F_{n}$ is finite and satisfies

$$
\# \operatorname{Fix}(\partial \alpha) \leq 4 n \text {. }
$$

It seems a natural question (posed originally to us by G Levitt) to ask whether automorphisms exist with trivial fixed subgroup which satisfy equality in this last formula, and if not, what the best possible bound is. In particular, one would like to 
know the answer to this question for the class of irreducible automorphisms $\alpha$ with irreducible powers (iwip), ie, $\alpha^{t}$ does not map any non-trivial proper free factor of $F_{n}$ to a conjugate of itself, for any $t \geq 1$. Since then, it has been shown by Guirardel [9] (see also Handel and Mosher [10]) that iwip automorphisms can never satisfy equality, see Remark 6.1.

In view of these results, this paper gives an answer to Levitt's question. We consider the following family of automorphisms:

$$
\begin{aligned}
\alpha_{n}: & F_{n} \\
a_{1} & \mapsto F_{n} \\
a_{2} & \mapsto a_{1} a_{2} \ldots a_{2} a_{1} a_{2} \\
a_{3} & \mapsto a_{3} a_{1} a_{2} a_{3} \\
& \vdots \\
a_{n} & \mapsto a_{n} a_{1} a_{2} a_{3} \ldots a_{n} .
\end{aligned}
$$

Theorem 1.1 For any $n \geq 3$ the automorphism $\alpha_{n}$ is irreducible with irreducible powers, has trivial fixed subgroup, and has precisely $4 n-1$ distinct fixed points at $\partial F_{n}$. Among these there are $2 n-1$ attractive ones and $2 n$ repelling ones. The same is true for all positive powers of $\alpha_{n}$.

The result and some related material will be discussed in the last section of this paper. Note also that an earlier version of this paper, containing already the main result, was ciculated as preprint in 1998.

\section{The attracting fixed points of $\partial \alpha_{n}$}

Consider the following set of $2 n-1$ infinite words, notice that they are all positive or negative and hence reduced, and check that they are fixed by $\alpha_{n}$. Here a positive (or a negative) word is a word in the given basis with only positive (or only negative) exponents. Similarly, a positive automorphism of $F_{n}$ is an automorphism for which the image of a given basis consists entirely of positive words in this basis.

$$
\begin{aligned}
& X_{1}=a_{1} a_{2} a_{3} \ldots a_{n} \alpha_{n}\left(a_{2} a_{3} \ldots a_{n}\right) \alpha_{n}^{2}\left(a_{2} a_{3} \ldots a_{n}\right) \alpha_{n}^{3}\left(a_{2} a_{3} \ldots a_{n}\right) \ldots \\
& X_{2}=a_{2} a_{1} a_{2} \alpha_{n}\left(a_{1} a_{2}\right) \alpha_{n}^{2}\left(a_{1} a_{2}\right) \alpha_{n}^{3}\left(a_{1} a_{2}\right) \ldots \\
& X_{3}=a_{3} a_{1} a_{2} a_{3} \alpha_{n}\left(a_{1} a_{2} a_{3}\right) \alpha_{n}^{2}\left(a_{1} a_{2} a_{3}\right) \alpha_{n}^{3}\left(a_{1} a_{2} a_{3}\right) \ldots \\
& X_{4}=a_{4} a_{1} a_{2} a_{3} a_{4} \alpha_{n}\left(a_{1} a_{2} a_{3} a_{4}\right) \alpha_{n}^{2}\left(a_{1} a_{2} a_{3} a_{4}\right) \alpha_{n}^{3}\left(a_{1} a_{2} a_{3} a_{4}\right) \ldots
\end{aligned}
$$




$$
\begin{aligned}
X_{n}= & a_{n} a_{1} a_{2} a_{3} \ldots a_{n} \alpha_{n}\left(a_{1} a_{2} a_{3} \ldots a_{n}\right) \alpha_{n}^{2}\left(a_{1} a_{2} a_{3} \ldots a_{n}\right) \\
& \alpha_{n}^{3}\left(a_{1} a_{2} a_{3} \ldots a_{n}\right) \ldots \\
Y_{2}= & a_{2}^{-1} a_{1}^{-1} a_{2}^{-1} \alpha_{n}\left(a_{1}^{-1} a_{2}^{-1}\right) \alpha_{n}^{2}\left(a_{1}^{-1} a_{2}^{-1}\right) \alpha_{n}^{3}\left(a_{1}^{-1} a_{2}^{-1}\right) \ldots \\
Y_{3}= & a_{3}^{-1} a_{2}^{-1} a_{1}^{-1} a_{3}^{-1} \alpha_{n}\left(a_{2}^{-1} a_{1}^{-1} a_{3}^{-1}\right) \alpha_{n}^{2}\left(a_{2}^{-1} a_{1}^{-1} a_{3}^{-1}\right) \ldots \\
Y_{4}= & a_{4}^{-1} a_{3}^{-1} a_{2}^{-1} a_{1}^{-1} a_{4}^{-1} \alpha_{n}\left(a_{3}^{-1} a_{2}^{-1} a_{1}^{-1} a_{4}^{-1}\right) \alpha_{n}^{2}\left(a_{3}^{-1} a_{2}^{-1} a_{1}^{-1} a_{4}^{-1}\right) \ldots \\
\vdots & \\
Y_{n}= & a_{n}^{-1} a_{n-1}^{-1} \ldots a_{2}^{-1} a_{1}^{-1} a_{n}^{-1} \alpha_{n}\left(a_{n-1}^{-1} \ldots a_{2}^{-1} a_{1}^{-1} a_{n}^{-1}\right) \\
& \alpha_{n}^{2}\left(a_{n-1}^{-1} \ldots a_{2}^{-1} a_{1}^{-1} a_{n}^{-1}\right) \ldots
\end{aligned}
$$

As all $\alpha_{n}\left(a_{i}\right)$ are positive and of length greater or equal to 2, it is easy to see that for any finite initial subword $X^{\prime}$ of $X_{i}$ (or of $Y_{i}$ ) the word $\alpha_{n}\left(X^{\prime}\right)$ is again an initial subword of $X_{i}$ (or of $Y_{i}$ ), and it is strictly longer. Hence all the above words define attractive fixed points of $\partial \alpha_{n}$, see [4, Section I]. From the sign of the exponents and from the initial letter it is easy to observe that they are pairwise distinct.

We will show in Section 4 that $\operatorname{Fix}\left(\alpha_{n}^{-1}\right)=\operatorname{Fix}\left(\alpha_{n}\right)=\{1\}$. Actually, we will show in Section $\mathrm{V}$ that there are non-trivial fixed and not even periodic conjugacy classes of $\alpha_{n}$. Hence, in view of the inequality from [4] stated in the Introduction, it could theoretically be that $\alpha_{n}$ or a power of $\alpha_{n}$ has one more attractive fixed point on $\partial F_{n}$. However, for a proper power of $\alpha_{n}$ this couldn't be the case, as then the whole $\alpha_{n}$-orbit of this point would be fixed, thus giving more attractive fixed points than the above inequality from [4] allows. For $\alpha_{n}$ itself this is excluded by the fact that there are only $2 n-1$ total occurences of any $a_{i}$ in any reduced word $\alpha_{n}\left(a_{i}\right)$, and this number is an upper bound for the number of Fix $\left(\alpha_{n}\right)$-orbits of attracting fixed points in $\partial F_{n}$, as has been shown in [2, Theorem 2] (where one uses of [4, Proposition 1.1] for translation into our terminology).

\section{The repelling fixed points of $\partial \alpha_{n}$}

In order to compute the inverse of $\alpha_{n}$ we first define iteratively $x_{0}=a_{1}^{-1}$ and, for any $k$ with $0 \leq k \leq n-1, x_{k+1}=a_{n-k} x_{k}^{2}$. We now notice that:

$$
\begin{array}{ll}
\alpha_{n}\left(x_{0}\right) & =\left(a_{1} a_{2} \ldots a_{n}\right)^{-1}, \\
\alpha_{n}\left(a_{n} x_{0}\right) & =a_{n}, \\
\alpha_{n}\left(x_{1}\right) & =\left(a_{1} a_{2} \ldots a_{n-1}\right)^{-1}, \\
\alpha_{n}\left(a_{n-1} x_{1}\right) & =a_{n-1},
\end{array}
$$




$$
\begin{array}{ll}
\alpha_{n}\left(x_{n-2}\right) & =\left(a_{1} a_{2}\right)^{-1}, \\
\alpha_{n}\left(a_{2} x_{n-2}\right) & =a_{2}, \\
\alpha_{n}\left(x_{n-1}\right) & =a_{1}^{-1}
\end{array}
$$

Hence $\alpha_{n}^{-1}$ is given by $a_{1} \mapsto x_{n-1}^{-1}, a_{n-k} \mapsto a_{n-k} x_{k}(k=0, \ldots, n-2)$. It is easy to see from the above computations that, if we replace the basis element $a_{1}$ by its inverse $a_{1}^{-1}=x_{0}$, one obtains $\alpha^{-1}$ again as positive automorphism, with respect to the new basis $\left\{x_{0}, a_{2}, a_{3}, \ldots, a_{n}\right\}$.

In order to describe the attractive fixed points of $\alpha_{n}^{-1}$, we need some further notation. Define

$$
\begin{aligned}
y_{k} & =x_{n-1} x_{k}^{-1} x_{0}^{-1} \quad(k=0, \ldots, n-2) \\
y & =x_{n-1} x_{0}^{-1} \\
z & =x_{0}^{-1} a_{n}^{-1} a_{n-1}^{-1} \ldots a_{2}^{-1} x_{n-1}
\end{aligned}
$$

and notice that these are all positive words in the above defined new basis. We now define the infinite words

$$
X_{k}=a_{n-k} x_{k} \alpha_{n}^{-1}\left(x_{k}\right) \alpha_{n}^{-2}\left(x_{k}\right) \alpha_{n}^{-3}\left(x_{k}\right) \ldots
$$

and

$$
Y_{k}=a_{n-k} x_{0}^{-1} y_{k}^{-1} \alpha_{n}^{-1}\left(y_{k}^{-1}\right) \alpha_{n}^{-2}\left(y_{k}^{-1}\right) \alpha_{n}^{-3}\left(y_{k}^{-1}\right) \ldots
$$

for $k=0, \ldots, n-2$, as well as

and

$$
\begin{gathered}
Y=x_{0}^{-1} y^{-1} \alpha_{n}^{-1}\left(y^{-1}\right) \alpha_{n}^{-2}\left(y^{-1}\right) \alpha_{n}^{-3}\left(y^{-1}\right) \ldots \\
Z=x_{0}^{-1} x_{n-1} \alpha_{n}^{-1}(z) \alpha_{n}^{-2}(z) \alpha_{n}^{-3}(z) \ldots
\end{gathered}
$$

We first compute that these words are all fixed by $\alpha_{n}^{-1}$ : For the $X_{k}$, the $Y_{k}$ and $Y$ this follows directly from the given definition of $\alpha_{n}^{-1}$, using in particular $\alpha_{n}^{-1}\left(x_{0}\right)=x_{n-1}$ and the definitions $(*)$. For $Z$ it follows from the following computation:

$$
\begin{aligned}
\alpha_{n}^{-1}\left(x_{0}^{-1} x_{n-1}\right) & =x_{n-1}^{-1} \alpha_{n}^{-1}\left(a_{2} \ldots a_{n}\right) x_{n-1} \alpha_{n}^{-1}(z) \\
& =x_{n-2}^{-2} a_{2}^{-1} \alpha_{n}^{-1}\left(a_{2}\right) \alpha_{n}^{-1}\left(a_{3} \ldots a_{n}\right) x_{n-1} \alpha_{n}^{-1}(z) \\
& =x_{n-2}^{-1} \alpha_{n}^{-1}\left(a_{3} \ldots a_{n}\right) x_{n-1} \alpha_{n}^{-1}(z) \\
& =x_{n-3}^{-2} a_{3}^{-1} \alpha_{n}^{-1}\left(a_{3}\right) \alpha_{n}^{-1}\left(a_{4} \ldots a_{n}\right) x_{n-1} \alpha_{n}^{-1}(z) \\
& =x_{n-3}^{-1} \alpha_{n}^{-1}\left(a_{4} \ldots a_{n}\right) x_{n-1} \alpha_{n}^{-1}(z) \\
& \vdots \\
& =x_{1}^{-1} \alpha_{n}^{-1}\left(a_{n}\right) x_{n-1} \alpha_{n}^{-1}(z) \\
& =x_{0}^{-1} x_{n-1} \alpha_{n}^{-1}(z)
\end{aligned}
$$

The fact that all these words are $\alpha_{n}^{-1}$-attracting is a direct consequence from the above observation that the words defined in $(*)$ are positive in the new basis. 
Observe next that these infinite words are pairwise distinct: The words $X_{k}$ and $Z$ are all eventually positive and start with a different letter (notice that the initial letter $x_{0}^{-1}$ of $Z$ is not cancelled), and the same is true for the remaining ones, which are all eventually negative. Notice however that, for $n=2$, the two words $Y_{0}$ and $Y$ are related by the equation

$$
Y_{0}=a_{2} x_{0}^{-1} a_{2}^{-1} x_{0} Y,
$$

and $a_{2} x_{0}^{-1} a_{2}^{-1} x_{0} \in \operatorname{Fix}\left(\alpha_{2}\right)=\operatorname{Fix}\left(\alpha_{2}^{-1}\right)$. In order to show that no such phenomenon occurs for $n \geq 3$ it will be proved in Section III that $\operatorname{Fix}\left(\alpha_{n}\right)=\operatorname{Fix}\left(\alpha_{n}^{-1}\right)$ is trivial. This implies, for $n \geq 3$, that $\partial \alpha_{n}^{-1}$ has $2 n$ attracting fixed points on $\partial F_{n}$ which are all in distinct $\operatorname{Fix}\left(\alpha_{n}^{-1}\right)$-orbits.

\section{The fixed subgroup of $\alpha_{n}$}

In order to determine the fixed subgroup of $\alpha_{n}$ we use the train track methods of Bestvina and Handel [1]. As $\alpha_{n}$ is positive, it follows that the standard rose $R_{n}$ with $n$ leaves admits a train track representative $f: R_{n} \rightarrow R_{n}$ of $\alpha_{n}$, given simply by realizing the words $\alpha_{n}\left(a_{k}\right)$ as reduced paths in $R_{n}$, with the unique vertex $*$ of $R_{n}$ as initial and terminal point.

Recall [1] that any conjugacy class [w] of $F_{n}$ fixed by the outer automorphism $\hat{\alpha}_{n}$ defined by $\alpha_{n}$ is represented in the train track representative $R_{n}$ by a loop $\gamma$ which is a concatenation of indivisible Nielsen paths (INP's). Hence, in order to show that Fix $\left(\alpha_{n}\right)=\{1\}$, it suffices to show that $f$ does not have any INP's. For this purpose we first check for illegal turns in $R_{n}$ : A straight forward inspection, comparing initial and terminal subwords of the $\alpha_{n}\left(a_{k}\right)$ reveals that there is only one illegal turn, given by $\left(\bar{a}_{1}, \bar{a}_{n}\right)$. Any INP in $R_{n}$ must be of the form $\gamma_{1} \gamma_{2}^{-1}$, such that $\gamma_{1}$ and $\gamma_{2}$ are legal paths which both have terminal point at $*$ and define there the above illegal turn. Hence one of the $\gamma_{i}$, say $\gamma_{1}$, ends in $a_{1}$, while the other one, $\gamma_{2}$, ends in $a_{n}$. Their $f$-images have to be legal paths of the form $f\left(\gamma_{1}\right)=\gamma_{1} \gamma_{3}$ and $f\left(\gamma_{2}\right)=\gamma_{2} \gamma_{3}$. Thus $\gamma_{3}$ ends in $a_{1} a_{2} \ldots a_{n}$.

Case $1 \gamma_{3}=a_{1} a_{2} \ldots a_{n}$. It follows that the second to last letter in $\gamma_{1}$, which preceeds $a_{1}$, has to have $\alpha_{n}$-image with terminal letter equal to $a_{1}$. But no such $a_{k}$ exists!

It follows that $\gamma_{3}$ ends in $a_{n} a_{1} a_{2} \ldots a_{n}$. Then the second to the last letter in $\gamma_{1}$, preceding $a_{1}$, must be $a_{n}$ or $a_{1}$.

Case $2 \gamma_{3}=a_{n} a_{1} a_{2} \ldots a_{n}$. Then in either of the last two subcases the last letter of $\gamma_{1}$ would have to be $a_{n-1}$, contradicting the above conditions. 
It follows that the second to last letter of $\gamma_{2}$ must be $a_{n-1}$, and that $\gamma_{3}$ ends in $a_{1} a_{2} \ldots a_{n} a_{1} a_{2} \ldots a_{n}$.

Case $3 \gamma_{3}=a_{1} a_{2} \ldots a_{n} a_{1} a_{2} \ldots a_{n}$. In this case the last letter of $\gamma_{2}$ is $a_{n-1}$, again contradicting the above conditions.

It follows that the second to last letter of $\gamma_{1}$ is not $\alpha_{n}$ but $a_{1}$, and the letter before must be $a_{n-1}$. At this point we know that $\gamma_{3}$ ends in $a_{n-1} a_{1} a_{2} \ldots a_{n} a_{1} a_{2} \ldots a_{n}$.

Case $4 \gamma_{3}=a_{n-1} a_{1} a_{2} \ldots a_{n} a_{1} a_{2} \ldots a_{n}$. Then the last letter of $\gamma_{1}$ would be $a_{n-2}$, contradicting the above conditions. It follows that the third to the last letter in $\gamma_{2}$ is $a_{n-2}$. But then the only one possibility left is:

Case $5 \gamma_{3}=a_{1} a_{2} \ldots a_{n-1} a_{1} a_{2} \ldots a_{n} a_{1} a_{2} \ldots a_{n}$. Here the last letter of $\gamma_{1}$ would be $a_{n-1}$, contradicting the above conditions.

Notice that the argument in case 4 requires $n \geq 3$.

This sweeps out all possibilities, and hence proves that there is no INP in $R_{n}$ with respect to the train track map $f$, for $n \geq 3$.

In Section $\mathrm{V}$ we will also consider the question of whether there exists a path $\gamma_{1} \gamma_{2}^{-1}$ in $R_{n}$ such that both $\gamma_{i}$ are legal, and $f\left(\gamma_{1}\right)=\gamma_{2} \gamma_{3}, f\left(\gamma_{2}\right)=\gamma_{1} \gamma_{3}$. The reader can check without much difficulty, following precisely the same cases as above, that such paths do not exist either.

\section{The irreducibility of $\alpha_{n}$}

If $\alpha_{n}$ or a positive power of it were reducible, then there would be a non-trivial proper free factor $F_{m}$ of $F_{n}$ which is left invariant (up to conjugation) by $\alpha_{n}^{t}$, for some $t \geq 1$. Passing over to an even higher power and restricting possibly to another proper free factor of $F_{m}$ we can then assume that either $\alpha_{n}^{t}$ induces the trivial outer automorphism on $F_{m}$, or else $\left.\alpha_{n}^{t}\right|_{F_{m}}$ is irreducible with irreducible powers. The first case is excluded by our results in Section $\mathrm{V}$, as then $\alpha_{n}$ would have at least one non-trivial periodic conjugacy class. To rule out the second case we have to apply the following irreducibility test, compare Bestvina and Handel [1] or Lustig [13, 14]:

Let $f: \Gamma \rightarrow \Gamma$ be a train track map in the sense of [1]. Replace every vertex $v$ in $\Gamma$ by the 1-skeleton of a $(k-1)$-simplex $\sigma(v)$, where $k$ is the number of edge gates at $v$. 
(Recall that two edge germs $d E$ and $d E^{\prime}$ raying out of a vertex $v$ belong to the same gate if and only if for some $t \geq 1$ the paths $f^{t}(E)$ and $f^{t}\left(E^{\prime}\right)$ have a non-tivial common initial subpath.) This replacement is done by glueing each such edge germ $d E_{i}$ to the vertex $v\left(d E_{i}\right)$ of $\sigma(v)$ which represents the gate to which $d E_{i}$ belongs. Now extend $f$ by mapping every edge $e$ of $\sigma(v)$ which connects $v\left(d E_{i}\right)$ to $v\left(d E_{j}\right)$ to the edge of $\sigma(f(v))$ which connects $v\left(f\left(d E_{i}\right)\right)$ to $v\left(f\left(d E_{j}\right)\right)$. If $f\left(d E_{i}\right)=f\left(d E_{j}\right)$, then map the whole edge $e$ to the vertex $v\left(f\left(d E_{i}\right)\right)$. Change the definition of $f$ along the edges of $\Gamma$ so that for any edge $E_{i}$ of $\Gamma$ the image is a reduced path in the new graph which agrees with the old $f\left(E_{i}\right)$ up to inserting precisely one of the "new" edges (ie, the ones from the 1-skeletons of the $(k-1)$-simplices $\sigma(v))$ between any two "old" edges which are adjacent in $f\left(E_{i}\right)$. This defines a new graph $\Gamma_{1}$ and a new map $f_{1}: \Gamma_{1} \rightarrow \Gamma_{1}$.

We now omit from $\Gamma_{1}$ all edges from the $(k-1)$-simplices $\sigma(v)$ which are not contained in any image $f_{1}^{t}\left(E_{i}\right)$, for any of the old edges $E_{i}$ and $t \geq 1$. Notice that this is done by a finite check, as $f_{1}$ is eventually periodic on the new edges. The resulting graph $\Gamma_{2}$ admits a self map $f_{2}=\left.f_{1}\right|_{\Gamma_{2}}: \Gamma_{2} \rightarrow \Gamma_{2}$, and it is easy to see that $f_{2}$ inherits from $f$ the properties of a train track map. Obviously there is a canonical map $\theta: \Gamma_{2} \rightarrow \Gamma$, defined by the inclusion $\Gamma_{2} \subset \Gamma_{1}$ and subsequent contraction of every new edge of $\Gamma_{1}$. Our definitions give directly $f_{2} \theta=\theta f$, up to possibly reparametrizing $f$ along the edges.

Proposition 5.1 (Irreducibility Criterion) Let $f: \Gamma \rightarrow \Gamma$ be a train track map in the sense of [1], assume that its transition matrix is irreducible with irreducible powers, and assume also that no $f_{*}^{t}$ with $t \geq 1$ fixes elementwise a proper free factor of $\pi_{1} \Gamma$, up to conjugacy. Then $f_{*} \in \operatorname{Out}\left(\pi_{1} \Gamma\right)$ is an irreducible automorphism with irreducible powers if and only if the induced map $\theta_{*}: \pi_{1} \Gamma_{2} \rightarrow \pi_{1} \Gamma$ on the fundamental groups is surjective.

Proof We freely use in this proof some of the $\mathbb{R}$-tree technology from [4] and from [14, Sections 3-5], from which we also borrow the terminology. In particular, we consider the $\alpha$-invariant $\mathbb{R}$-tree $T$ with stretching factor $\lambda>1$ which is given by the (up to scalar multiples) well defined Perron-Frobenius row eigen vector $\vec{v}_{*}$ of the transition matrix $M(f)$ of the train track map $f$. It comes with an $F_{n}$-equivariant map $i: \tilde{\Gamma} \rightarrow T$ which is isometric on edges (and more generally on legal paths), if the universal covering $\tilde{\Gamma}$ is provided with edge lengths as given by $\vec{v}_{*}$. Furthermore, there is a homothety $H: T \rightarrow T$ with stretching factor $\lambda$, which $\alpha$-twistedly commutes with the $F_{n}$-action: It satisfies $\alpha(w) H=H w: T \rightarrow T$ for all $w \in F_{n}$. If $\tilde{f}$ is the lift of $f$ to $\tilde{\Gamma}$ that also $\alpha$-twistedly commutes with the $F_{n}$-action, then $H$ and $\tilde{f}$ commute via $i$, ie, $H i=i \tilde{f}$. 
We now assume that the map $\theta$ is not surjective, ie, some of the 1 -skeleta $\sigma^{1}(v)$ of the simplices $\sigma(v)$ decompose into more than one connected component, when passing from $\Gamma_{1}$ to $\Gamma_{2}$. We pass over to a new graph $\Gamma_{3}$ in the following way:

For each of the simplices $\sigma(v)$ we reconnect the connected components of $\sigma^{1}(v) \cap \Gamma_{2}$ by adding a new center vertex $c(v)$ to $\Gamma_{2}$ and connecting each connected component by a central edge to $c(v)$. We extend the train track map $f_{2}$ canonically to obtain again a train track map $f_{3}: \Gamma_{3} \rightarrow \Gamma_{3}$, and a "projection map" $\theta_{3}: \Gamma_{3} \rightarrow \Gamma$ with $\theta_{3} f_{3}=f \theta_{3}$ (up to isotopy within the images of single edges). By construction, $\theta_{3 *}$ is now surjective. Note that the map $f_{3}$ respects the partition of the edges of $\Gamma_{3}$ into edges from $\Gamma_{2}$ and central edges.

We consider the universal covering $\tilde{\Gamma}_{3}$ and the canonical $F_{n}$-equivariant map $i_{3}: \tilde{\Gamma}_{3} \rightarrow$ $T$ obtained from composing the lift of $\theta_{3}$ to $\tilde{\Gamma}$ with the above map $i$. Just as for $\Gamma$ we can also consider the transition matrix for $f_{3}$ and obtain in the analogous way Perron-Frobenius edge lengths on $\tilde{\Gamma}_{3}$ to make the map $i_{3}$ edge isometric. Of course, the resulting "metric" on $\tilde{\Gamma}_{3}$ is only a pseudo-metric, as all of the newly introduced central edges will get Perron-Frobenius length 0 .

The usefulness of these "invisible" central edges however becomes immediately appearent: Each multipod $Y(\tilde{v})$, consisting of the lift to $\tilde{\Gamma}_{3}$ of a central vertex $c(v)$ with all adjacent central edges, is mapped by $i_{3}$ to a single point $Q(\tilde{v})=i(\tilde{v})$ in $T$ (here $\tilde{v} \in \tilde{\Gamma}$ is the corresponding lift of the vertex $v \in \Gamma$ ), and the directions at this point are in canonical bijection (given by the map $i_{3}$ ) with the gates at $\tilde{v}$ and hence with the endpoints of the multipod $Y(\tilde{v})$. We can $F_{n}$-equivariantly replace the point $Q(\tilde{v})$ by the multipod $Y(\tilde{v})$, where every direction of $T$ at $Q(\tilde{v})$ is attached at the corresponding endpoint of $Y(\tilde{v})$. Again, we define the edge lengths throughout $Y(\tilde{v})$ to be 0 , so that metrically the resulting tree $T_{3}$ is the same as $T$.

We now observe that the homothety $H_{3}: T_{3} \rightarrow T_{3}$, which $T_{3}$ canonically inherits from $H: T \rightarrow T$, can be shown to map on one hand the union $Y$ of all $Y(\tilde{v})$ to itself, but similarly also its complement $T_{3} \backslash Y$. This follows from the commutativity equality $i_{3} \tilde{f}_{3}=H_{3} i_{3}$ which is by the above construction inherited from the equation $i \tilde{f}=H i$, and from the above observation that the subgraph $\Gamma_{2}$ of $\Gamma_{3}$, as well as its complement $\Gamma_{3} \backslash \Gamma_{2}$, is kept invariant under the map $f_{3}$. As a consequence, we can invert the situation, by considering the length function (also a row eigen vector of $M\left(f_{3}\right)$ !) which associates length 1 to every edge of $Y$, and length 0 to all other edges, ie, contracting every complementary component of $Y$ in $T_{3}$ to a point. The resulting space $T_{3}^{*}$ is a simplicial $\mathbb{R}$-tree with trivial edge stabilizers, and the map $\mathrm{H}_{3}$ induces an isometry $H_{3}^{*}: T_{3}^{*} \rightarrow T_{3}^{*}$ which $\alpha$-twistedly commutes with $\tilde{f}$ and commutes with the induced 
map $i_{3}^{*}: \tilde{\Gamma}_{3} \rightarrow T_{3}^{*}$. It follows that the Bass-Serre decomposition of $F_{n}$ associated to this simplicial tree is $\alpha$-invariant. In particular, the vertex groups of this decomposition give a non-empty collection of non-trivial proper free factors of $F_{n}$ which is $\alpha$-invariant, proving directly that $\alpha$ is not iwip.

To prove the converse implication of the theorem we can now invert every step in the construction given above: If $\alpha$ is reducible and no positive power fixes elementwise a free factor, there exists a simplicial tree as $T_{3}^{*}$, and this tree is given (compare [4]) by a row eigenvector for the top stratum of some relative train track representative $f_{0}: \Gamma_{0} \rightarrow \Gamma_{0}$ of $\alpha$ as in [1]. Modifying this train track representative as in [13] to get a partial train track representative with Nielsen faces $\phi: \mathcal{G} \rightarrow \mathcal{G}$, allows us, as above for the graph $\Gamma_{3}$, to represent simultaneously both, the action on $T_{3}^{*}$ as well as that on $T$, by row eigen vectors of $M(\phi)$. As a consequence one sees that the two trees come from a common "refinement", as given above by the tree $T_{3}$ : Both, $T_{3}^{*}$ and $T$, are defined by a pseudo-metric on $T_{3}$ which is troughout zero, on vice-versa complementary $H_{3}$-invariant subforests of $T_{3}$. We now consider again the originally given train track map $f: \Gamma \rightarrow \Gamma$ and its local "blow-up" $f_{1}: \Gamma_{1} \rightarrow \Gamma_{1}$. The $H_{3}$-invariance of the two subforests translates (via the map $i_{1}: \tilde{\Gamma}_{1} \rightarrow T$ induced by $i$ ) into a non-trivial $f_{1}$-invariant subgraph of the union of the $\sigma^{1}(v)$, with invariant complement $\Gamma_{2}$. The connected components of this graph $\Gamma_{2}$ are in 1-1 correcpondence with the $F_{n}$-orbits of the zero-valued subforests of $T_{3}$ defined by the row-eigen vector that gives $T_{3}^{*}$. Thus the non-triviality of the latter translates directly into the fact that the injection $\pi_{1} \Gamma_{2} \rightarrow \pi_{1} \Gamma_{1}$ is non-surjective. This finishes the proof.

Remark 5.2 The Irreducibility Criterion (Proposition 5.1) can alternatively be derived as consequence of the theory of limit laminations and their fundamental group, as developed in [12]. We sketch now an outline of the "if"-direction:

Reducibility of $f_{*}$ or some positive power would give, as above explained for $\alpha_{n}$, a proper free factor $F_{m}$ of $F_{n}$ on which $f_{*}^{t}$ for some $t \geq 1$ acts as irreducible automorphism with irreducible powers. Such an automorphism has an expanding limit lamination $L$ with $\pi_{1} L \subset F_{m}$. As $F_{m}$ embeds as free factor into $F_{n}$, say $\rho: F_{m} \rightarrow F_{n}$, we obtain $\pi_{1}(\rho(L)) \subset \rho\left(F_{m}\right) \neq F_{n}$ (compare [12, Lemma 9.7]). On the other hand, it follows from the irreducibility of the transition matrix of $f: \Gamma \rightarrow \Gamma$ that there is only one expanding limit lamination $L^{\infty}(f)$. Hence $L^{\infty}(f)=\rho(L)$, and we can apply [12, Korollar 7.7] with $\tau=\Gamma_{2}$ (provided with an appropriate combinatorial labeling which reflects $\theta_{*}$ ) to deduce $\pi_{1}\left(L^{\infty}(f)\right)=F_{n}$ from the surjectivity of $\theta_{*}$, thus yielding a contradiction to the above derived statement $\pi_{1}(\rho(L)) \subset \rho\left(F_{m}\right) \neq F_{n}$.

In order to apply the Irreducibility Criterion 5.1 to the automorphism $\alpha_{n}$ as given in 
the Introduction, we first compute directly from the definition of the $\alpha_{n}\left(a_{i}\right)$ that the transition matrix of $f$ is irreducible with irreducible powers. Then we have to replace the vertex $*$ by part of the 1 -skeleton of a $(2 n-1)$-simplex $\sigma=\sigma(*)$. We start with the 0 -skeleton, and introduce only those edges of $\sigma$ which are contained in the $f_{1}$-image of any of the old edges. This gives two connected components, where one of them contains only the vertex associated to the initial germ of $a_{2}$ and the one associated to the terminal germ of $a_{1}$, as well as a single new edge, say $\eta$, which connects them. The other component contains all other vertices and a tree which connects them (with the vertex asociated to the initial germ of $a_{1}$ as "root" of the tree). Now we have to fill in the forward $f_{1}$-orbit of the new edges introduced so far. But the $f_{1}$-image of $\eta$ connects the vertex of the initial germ of $a_{2}$ to that of the terminal germ of $a_{n}$, so that in $\Gamma_{2}$ the subgraph which belongs to the $(2 n-1)-\operatorname{simplex} \sigma$ is connected. Hence $\theta_{*}$ is surjective.

\section{End of the proof and some remarks}

In this section we consider the outer automorphism $\hat{\alpha}_{n}$ induced by $\alpha_{n}$, and its inverse $\hat{\alpha}_{n}^{-1}$. In [4] an index for outer automorphisms of $F_{n}$ has been defined as follows: Two automorphisms of $F_{n}$ are called isogredient (or in [4] similar), if they are conjugated in $\operatorname{Aut}\left(F_{n}\right)$ by an inner automorphism of $F_{n}$. Let $\mathcal{S}(\hat{\alpha})$ denote the set of isogredience classes $\left[\alpha^{\prime}\right]$ of automorphisms $\alpha^{\prime}$ inducing the outer automorphism $\hat{\alpha}^{\prime}=\hat{\alpha}$. We define

$$
\operatorname{ind}(\hat{\alpha}):=\sum_{\left[\alpha^{\prime}\right] \in \mathcal{S}(\hat{\alpha})} \max \left(\operatorname{rk}\left(\operatorname{Fix}\left(\alpha^{\prime}\right)\right)+\frac{1}{2} \#\left(\operatorname{Fix}^{+}\left(\partial \alpha^{\prime}\right) / \operatorname{Fix}\left(\alpha^{\prime}\right)\right)-1,0\right) .
$$

The main result of $\left[4\right.$, Theorem $\left.1^{\prime}\right]$, is equivalent to the inequality

$$
\operatorname{ind}(\hat{\alpha}) \leq n-1
$$

for all $\hat{\alpha} \in \operatorname{Out}(F)$.

Now, the outer automorphism $\hat{\alpha}_{n}^{-1}$ has maximal possible index $n-1$, all concentrated in one isogredience class of $\hat{\alpha}_{n}^{-1}$, namely the one given by $\alpha_{n}^{-1}$, and here again all concentrated in the term $\frac{1}{2} \#\left(\operatorname{Fix}^{+}\left(\partial \alpha_{n}^{-1}\right) / \operatorname{Fix}\left(\alpha_{n}^{-1}\right)\right)$, which counts the number of the attractive fixed points at $\partial F_{n}$, as the fixed subgroup of $\alpha_{n}^{-1}$ is trivial.

We remark at this point that, if $X$ and $Y$ are infinite words, both fixed by an automorphism $\alpha$, and $w X=Y$ for some $w \in F_{n}$, then it follows from an elementary combinatorial case checking that $\alpha(w)=w$. Hence we know that the index contribution of the $2 \mathrm{n}$ attracting fixed points of $\alpha_{n}^{-1}$ computed in Section II will be the same for all positive 
powers of $\alpha_{n}^{-1}$ : On the other hand (compare [1]), a fixed non-trivial conjugacy class for some $\alpha_{n}^{-t}, t \geq 1$, will be represented by a concatenation of INP's of a train track representative of $\alpha_{n}^{-t}$, which would contribute at least one infinite attracting fixed word in the same isogredience class of $\hat{\alpha}_{n}^{-t}$ which fixes the non-trivial word read off from the concatenation of INP's. Hence we would get another positive index contribution for $\hat{\alpha}_{n}^{-t}$, in contradiction to the above inequality for the index. Hence $\alpha^{-1}$ and thus $\alpha$ can not have non-trivial periodic conjugacy classes.

Remark 6.1 Outer automorphisms of $F_{n}$ with a positive power of index $n-1$ which are not geometric (ie, they are not induced by a homeomorphisms of a surface with boundary) have been termed para-geometric in [4, Section VI], as, just as for geometric automorphisms, their action on any forward limit tree is geometric (in the sense of Gaboriau and Levitt [5]). Guirardel [9] shows that if for an iwip automorphism $\alpha$ both, the (uniquely determined) forward and the backward limit trees are geometric ( $\Longleftrightarrow \operatorname{ind}\left(\hat{\alpha}^{t}\right)=\operatorname{ind}\left(\hat{\alpha}^{-t}\right)=n-1$ for some sufficiently large $t \geq 1$ ), then $\alpha$ is geometric.

To see whether the irreducible (and non-geometric !) automorphism $\alpha_{n}$ itself is parageometric or not we can either apply the result of Guirardel [9] quoted in the Introduction, or else apply direct arguments which seem interesting in their own right, as they are typical for similar computations for many other automorphisms:

We will compute the index of $\alpha_{n}$ and that of its positive powers: From the previous sections we know already that there is one isogredience class, given by $\alpha_{n}$, which contributes 0 from $\operatorname{Fix}\left(\alpha_{n}\right)$ and $2 n-1$ from $\operatorname{Fix}^{+}\left(\partial \alpha_{n}\right)$, adding properly up to an index contribution of $\operatorname{rk}\left(\operatorname{Fix}\left(\alpha_{n}\right)\right)+\frac{1}{2} \#\left(\operatorname{Fix}^{+}\left(\partial \alpha_{n}\right) / \operatorname{Fix}\left(\alpha_{n}\right)\right)-1=n-\frac{3}{2}$. Hence the only possibility for $\hat{\alpha}_{n}$ to have index $n-1$ is if there is another isogredience class, represented by some automorphism $\alpha_{n}^{\prime}$, with index contribution of $\frac{1}{2}$. As we have shown above that there is no non-trivial conjugacy class fixed by $\hat{\alpha}_{n}$, the only possibility is that this $\alpha_{n}^{\prime}$ has 3 attracting fixed points at $\partial F_{n}$. In this case the train track representative $f: R_{n} \rightarrow R_{n}$ of $\hat{\alpha}_{n}$ has to have either another fixed point with 3 distinct fixed directions (= edge germs), but this is not the case as $R_{n}$ has only one vertex. Otherwise there must be two distinct fixed points in $R_{n}$, each with 2 fixed directions, and they are connected by an INP. But we have shown in Section III that INP's do not exist for $f: R_{n} \rightarrow R_{n}$. Hence it follows that ind $\left(\hat{\alpha}_{n}\right)=n-\frac{3}{2}$.

The same arguments apply to all positive powers of $\alpha_{n}$, except that we have to rule out also the possibility of periodic INP's: If there is an INP for $\alpha_{n}^{t}$ which is not an INP for $\alpha_{n}$, then its whole $\alpha_{n}$-orbit consists of INP's for $\alpha_{n}^{t}$. As this would immediately give 
a too large index for $\hat{\alpha}_{n}^{t}$ if the orbit consists of more than one INP, the only possibility left is that there is an INP for $\alpha_{n}^{2}$, and $\alpha$ fixes this path too, but reverses its orientation. But this possibility has been ruled out in the last paragraph of Section III. Thus $\alpha_{n}$ is not parageometric (and also not geometric).

To finish this discussion, we would like to point out a subtle point in which the non-geometric and non-parageometric $\alpha_{n}$ and the parageometric $\alpha_{n}^{-1}$ differ, which is characteristic for their classes:

For any parageometric automorphism (as $\hat{\alpha}_{n}^{-1}$ ) there is a stable train track representative with a single illegal turn, namely the one at the tip of the unique INP, see [1]. If we keep folding at this illegal turn, we get iteratively smaller and smaller copies of the train track, thus realizing the inverse of the train track map by "continuous iterated folding" (compare [11]). Now, if we consider the train track representative $f: R_{n} \rightarrow R_{n}$ of the (non-parageometric !) $\hat{\alpha}_{n}$ from Section III, there is also a single illegal turn, and if we keep folding there, it turns out that this will always be the case, as there will never appear any other illegal turn. Thus the situation looks remarkably similar to that in the parageometric case. There is, however, an interesting difference: If we trace in $R_{n}$ (or rather in the universal covering $\tilde{R}_{n}$ ) the two "paths" which will be folded together in this iterative folding procedure, we will see that these are not two continuous arcs with the same initial point (as would be true in the parageometric case, given there by the two subarcs of the INP which meet at the illegal turn), but much rather there will be lots of (indeed infinitely many !) discontinuities in these "paths". Each of these will disappear eventually in the folding process, but initially they are present. We believe that in these discontinuities the core information is encoded, for a geometric understanding of the gap between the maximal index of a positive power of the automorphism and the above upper bound $n-1$.

\section{References}

[1] M Bestvina, M Handel, Train tracks and automorphisms of free groups, Ann. of Math. (2) 135 (1992) 1-51 MR1147956

[2] MM Cohen, M Lustig, On the dynamics and the fixed subgroup of a free group automorphism, Invent. Math. 96 (1989) 613-638 MR996557

[3] D Cooper, Automorphisms of free groups have finitely generated fixed point sets, J. Algebra 111 (1987) 453-456 MR916179

[4] D Gaboriau, A Jaeger, G Levitt, M Lustig, An index for counting fixed points of automorphisms of free groups, Duke Math. J. 93 (1998) 425-452 MR1626723 
Free group automorphisms with many fixed points at infinity

[5] D Gaboriau, G Levitt, The rank of actions on R-trees, Ann. Sci. École Norm. Sup. (4) 28 (1995) 549-570 MR1341661

[6] D Gaboriau, G Levitt, M Lustig, A dendrological proof of the Scott conjecture for automorphisms of free groups, Proc. Edinburgh Math. Soc. (2) 41 (1998) 325-332 MR1626429

[7] S M Gersten, Fixed points of automorphisms of free groups, Adv. in Math. 64 (1987) 51-85 MR879856

[8] R Z Goldstein, E C Turner, Fixed subgroups of homomorphisms of free groups, Bull. London Math. Soc. 18 (1986) 468-470 MR847985

[9] V Guirardel, Cour et nombre d'intersection pour les actions de groupes sur les arbres, Ann. Sci. École Norm. Sup. (4) 38 (2005) 847-888 MR2216833

[10] M Handel, L Mosher, Parageometric outer automorphisms of free groups, Trans. Amer. Math. Soc. 359 (2007) 3153-3183 MR2299450

[11] J Los, M Lustig, The set of train track representatives of an irreducible free group automorphism is contractible Available at http://www.crm.es/Publications/04/pr606.pdf

[12] M Lustig, Automorphismen von freien Gruppen, Habilitationsschrift, Ruhr-Universität Bochum (1992)

[13] M Lustig, Structure and conjugacy for automorphisms of free groups I, II, Max-Planck Institut für Mathematik, Preprint Series 2000 (No 130) and 2001 (No 4) Available at http://www.mpim-bonn.mpg.de

[14] M Lustig, Conjugacy and centralizers for iwip automophisms of free groups, from: “Geometric Group Theory”, Trends in Mathematics, Birkhäuser (2007) 197-224

[15] F Paulin, Sur les automorphismes extérieurs des groupes hyperboliques, Ann. Sci. École Norm. Sup. (4) 30 (1997) 147-167 MR1432052

Louis Pasteur-Strasse 58, 60439 Frankfurt am Main, Germany

Mathématiques (LATP), Université P Cézanne - Aix Marseille III

Ave E Normandie-Niemen, 13397 Marseille 20, France

Andre.Jaeger@ui-gmbh.de, Martin.Lustig@univ-cezanne.fr

Received: 27 June $2006 \quad$ Revised: 5 July 2007 